\title{
Considerations on cardiac patients during Covid-19 outbreak
}

The ongoing coronavirus disease (Covid-19) pandemic has challenged globalized society to cope with the adoption of revolutionary healthcare measures. The severe acute respiratory syndrome coronavirus-2 (SARS-CoV-2) not only causes viral pneumonia but also acute myocardial injury and chronic damage to the cardiovascular system. ${ }^{1}$ Currently, treating patients with cardiovascular disease (CVD) has become more challenging. A network of "hub" and "spoke" centers based on a system of specialized Covid-19 referral hospitals has been organized, in order to guarantee optimal medical care for patients with cardiac and noncardiac emergencies. Indeed, in Lombardy, Italy (the epicenter of the European outbreak), the STelevation myocardial infarction (STEMI) regional network has been rearranged, reducing by more than $75 \%$ the number of previous "hub" centers with 24 hours a day-7 days a week capacity to perform primary percutaneous coronary interventions (PCI), with 13 hospitals acting as "hubs" and other 42 acting as "spokes". ${ }^{2}$ Generally, we have witnessed a drastic reduction of patients referring nonCovid-19 related symptoms to the emergency department. The fear of contagion has caused a significant drop in improper admissions to the hospital facilities. In particular, national and international registries have reported a significant decrease in the number of admissions for acute coronary syndromes (ACS), especially in northern Italy. Realistically, more and more people with ACS have not sought medical attention and have been left untreated, as it is confirmed by the increasing delayed presentations of ACS patients and their higher mortality. Therefore, an alarming increase in worse ACS outcomes is expected in the near future.

The issue is amplified by the uncertain trend of this pandemic and by the unpredictable duration of the lockdown in some countries. Moreover, the limited mobility measures and the weak integration between hospitals and territorial medicine, especially in high-risk areas, constitute an additional concern. The most vulnerable Covid19-free subjects, such as patients with chronic cardiac disorders (ie, heart failure), have not routinely been followed-up in the hospital facilities during the pandemic. A rapid reorganization of cardiac services and practical guidance on how to manage chronic patients are needed in the shortest time. Telemedicine and telecardiology, integrated with the traditional management, appear to be precious tools for this emergent medical model, focused on the interplay between social, economic, environmental, and clinical factors. The flexible use of telematic devices, now available for teleconsultation and/or remote monitoring, allows the creation of integrated and personalized management programs that are effective and efficient. Indeed, in order to minimize risks of in-hospital SARS-CoV-2 spreading, telemedicine should be adopted whenever possible, especially for frail and older patients. Telemedicine is crucial during this viral outbreak, preventing patients' health from deteriorating because of mistreated CVD while coping with the high infectious risks. ${ }^{3}$ Furthermore, telerehabilitation should be considered as an option for patients discharged from hospitals after an ischemic event and healthcare providers should be aware of innovative program delivery models. Both European and American Societies have shown to be up to the challenge, providing statements and recommendations on how to manage cardiac patients in these critical times, ${ }^{4,5}$ and, more importantly, encouraging the rapid sharing of clinical experience and knowledge through any available sources (eg, webinars, free online access to nearly all medical resources).

One of the most burning issues has been the appropriate use of cardiovascular imaging in diagnosis and management of both Covid-19 and non-Covid-19 patients. It has become widely accepted that traditional cardiovascular risk factors, particularly older age, previous CVD, diabetes, and hypertension, increase significantly the risk of mortality in Covid-19 patients. ${ }^{1}$ Therefore, indications and recommended tests to assess cardiovascular function in suspected or confirmed Covid-19 cases need to be standardized. The shared principles at the base of cardiovascular imaging are appropriateness of indication and diagnostic yield of the test, as well as choosing the most suitable, shortest and safest examination, both for patients and healthcare personnel. Transthoracic echocardiography might be very useful in this setting, since it allows a bedside cardiac and respiratory assessment and remote assistance if needed, but unfortunately requires close contact with the patient. Therefore, Focused Cardiac Ultrasound Study (FoCUS), Ultrasound Assisted Physical Examination (UAPE), and Point Of Care Cardiac Ultrasound (POCUS), in accordance with American Society of Echocardiography, are recommending to reduce the duration of healthcare workers exposure and to limit the use of additional resources, such as personal protective equipment (PPE). During this outbreak, special attention has to be dedicated to PPE, which has to be adequate to the risks (low/minimal: not suspected; moderate: suspected; high: confirmed), that should be evaluated before starting the examination. Therefore, careful planning and review of medical history are crucial to obtain complete diagnostic views, in order to avoid the need of subsequent additional images. Because of the high risk of aerosol generating procedures, transesophageal echocardiography should be replaced with alternative imaging modalities whenever possible. Computed tomography (CT) has gained attention, not only for its crucial role in confirming Covid-19 pneumonia, but also for the possible synergies and opportunities in evaluating cardiac morphology. Coronary CT showed to be accurate in the evaluation of both chronic coronary 
syndromes and $\mathrm{ACS}^{6,7}$ and thus can provide noninvasive informations to solve doubtful situations, for example, unexplained elevation of cardiac troponins, (frequent finding in Covid-19 patients).

Cardiac magnetic resonance (CMR) may be provided also for urgent care in patients who have no known active Covid-19. When necessary, CMR in patients with confirmed or suspected SARSCoV-2 infections should be focused on the specific clinical question with an emphasis on myocardial function and tissue characterization while optimizing patients and staff safety. CMR have had a marginal role so far during this pandemic, due to the long duration of the examinations and its limited availability. Nevertheless, in patients with confirmed SARS-CoV-2 infections and underlying CVD or myocardial injury, indications for CMR may evolve. Indeed, CMR may offer an effective choice to obtain critical information for clinical decision-making, being the gold standard for evaluation of cardiac function and tissue characterization. If cardiovascular imaging in Covid-19 patients could be challenging, the management of nonCovid patients may be even more complicated. Both American and European Societies of Cardiology recommend the deferral or even the cancellation of elective nonurgent and routine follow-up visits, encouraging the use of telemedicine, in order to avoid SARS-CoV-2 spreading and to protect healthcare workers. However, the triage of patients with CVD is seldom black or white: Many patients could overlook signs and symptoms of clinical worsening when reporting to physicians. Moreover, SARS-CoV-2 infections share symptoms with ACS, pulmonary embolism, and acute heart failure, like shortness of breath, fatigue, weakness, and sometimes chest pain.

\section{1 | CONCLUSIONS}

Severe acute respiratory syndrome coronavirus- 2 infection has major implications for the cardiovascular system. Given the decrease in new Covid-19 cases worldwide, now, we are approaching the so-called "Phase 2" that represents a challenge that is leading to a gradual return to pre-Covid-19 life. The epidemiological and clinical situation is rapidly evolving and routine clinical practice depends on institutions and local availability. We expect a continue and progressive update of the recommendations, which serve as general framework in cardiac patients.
Fabiola B. Sozzi MD, $\mathrm{PhD}^{1}$

Marta Belmonte $\mathrm{MD}^{1}$

Marco Schiavone $\mathrm{MD}^{1}$ iD

Ciro Canetta $\mathrm{MD}^{2}$

Francesco Blasi MD, FERS ${ }^{1}$

${ }^{1}$ Fondazione Ospedale Maggiore Policlinico IRCCS Cà Granda, Milan, Italy

${ }^{2}$ Ospedale di Crema, Lombardy, Italy

Correspondence

Fabiola B. Sozzi, Ospedale Maggiore Policlinico IRCCS, Milan,

Italy.

Email: fabiola_sozzi@yahoo.it

\section{ORCID}

Fabiola B. Sozzi (iD https://orcid.org/0000-0002-2153-6256

Marco Schiavone iD https://orcid.org/0000-0003-0720-3380

\section{REFERENCES}

1. Guo T, Fan Y, Chen M, et al. Cardiovascular implications of fatal outcomes of patients with coronavirus disease COVID-19. JAMA Cardiol. 2020; e201017.https://doi.org/10.1001/jamacardio.2020.1017. Online ahead of print

2. Stefanini GG, Azzolini E, Condorelli G. Critical organizational issues for cardiologists in the COVID-19 outbreak: a frontline experience from Milan, Italy. Circulation. 2020;141(20):1597-1599.

3. Hollander JE, Carr BG. Virtually perfect? Telemedicine for Covid-19. N Engl J Med. 2020;382(18):1679-1681.

4. ESC guidance for the diagnosis and management of CV disease during the COVID-19 pandemic. https://www.escardio.org/Education/ COVID-19-and-Cardiology/ESC-COVID-19-Guidance. Last accessed June 8, 2020.

5. ASE Statement on COVID-19. https://www.asecho.org/ase-state ment-covid-19/. Last accessed June 8, 2020.

6. Knuuti J, Wijns W, Saraste A, et al. ESC Guidelines for the diagnosis and management of chronic coronary syndromes: the task force for the diagnosis and management of chronic coronary syndromes of the European Society of Cardiology (ESC). Eur Heart J. 2020;41(3):407-477.

7. Nieman K, Hoffmann U. Cardiac computed tomography in patients with acute chest pain. Eur Heart J. 2015;36(15):906-914. 